Миграция спицы Киршнера в позвоночный канал после фиксации акромиально-ключичного сочленения (обзор литературы и клиническое наблюдение)

\author{
Д.А. Гуляев ${ }^{1}$, Д.С. Годанюк ${ }^{1}$, Т.А. Каурова ${ }^{1}$, П.В. Красношлык ${ }^{1}$, С.В. Майков ${ }^{2}$ \\ ${ }^{1}$ ФГБУ «Национальный медицинский исследовательский центтр им. В.А. Алмазова» Минздрава России, \\ Санкт-Петербург, Россия \\ ${ }^{2}$ ФГБУ «Российский научно-исследовательский институт травматологии и ортопедии им. Р.Р. Вредена» \\ Минздрава России, Санкт-Петербург, Россия
}

\title{
Реферат
}

Перелом и миграция элементов металлоконструкций являются широко известной проблемой, актуальность которой особенно высока в активно нагружаемых зонах с высокой амплитудой физиологических движений. Для верхнего плечевого пояса и области шеи характерно наличие большого количества критически важных сосудистых и нервных образований, что делает миграцию имплантатов в этих анатомических зонах жизнеугрожающим осложнением. Миграция имплантов в позвоночный канал является редким осложнением, поэтому тактика ведения подобных пациентов освещена недостаточно. В статье приводится обзор 17 сообщений, посвященных миграции имплантов в позвоночный канал после фиксации акромиально-ключичного сочленения спицами Киршнера. Обобщены представления о причинах миграции, хирургической тактике, профилактические рекомендации. Основной причиной перелома и миграции спиц Киршнера при фиксации повреждений акромиально-ключичного сочленения является нестабильность конструкции, трансартикулярное проведение спиц при фиксации устраненного вывиха акромиального конца ключицы, недостаточность иммобилизации и несвоевременное удаление конструкций после снятия иммобилизации. Миграция элементов конструкции в позвоночный канал является показанием для их хирургического удаления вне зависимости от клинических проявлений. В большинстве изученных сообщений авторы использовали задние или соосные с направлением миграции латеральные доступы. Способы пластики дефектов твердой мозговой оболочки в изученной литературе не приводятся. Авторы приводят собственное клиническое наблюдение миграции фрагмента спицы Киршнера в позвоночный канал у пациента спустя 10 лет после фиксации акромиально-клинического сочленения. На основании обзора литературы и анализа рисков основных этапов операции приводится обоснование выбранной тактики удаления мигрировавшего имплантата с использованием комбинации заднего и бокового доступов.

Ключевые слова: миграция спицы Киршнера, повреждение спинного мозга, ликворея, акромиальноключичное сочленение, перелом ключицы.

\section{Kirschner Wire Migration into Spinal Canal after Acromioclavicular Joint Fixation (Literature Review and Clinical Case)}

\author{
D.A. Gulyaev ${ }^{1}$, D.S. Godanyuk ${ }^{1}$, T.A. Kaurova ${ }^{1}$, P.V. Krasnoshlyk ${ }^{1}$, S.V. Maikov ${ }^{2}$ \\ ${ }^{1}$ Almazov National Medical Research Centre, St. Petersburg, Russian Federation \\ ${ }^{2}$ Vreden Russian Research Institute of Traumatology and Orthopedics, St. Petersburg, Russian Federation
}

미 Гуляев Д.А., Годанюк Д.С., Каурова Т.А., Красношлык П.В., Майков С.В. Миграция спицы Киршнера в позвоночный канал после фиксации акромиально-ключичного сочленения (обзор литературы и клиническое наблюдение). Травматология и ортопедия России. 2018;24(4):121-128. DOI: 10.21823/2311-2905-2018-24-4-121-128.

Cite as: Gulyaev D.A., Godanyuk D.S., Kaurova T.A., Krasnoshlyk P.V., Maikov S.V. [Kirschner Wire Migration into Spinal Canal after Acromioclavicular Joint Fixation (Literature Review and Clinical Case)]. Travmatologiya $i$ ortopediya Rossii [Traumatology and Orthopedics of Russia]. 2018;24(4):121-128. (In Russ.). DOI: 10.21823/2311-2905-2018-24-4-121-128.

Годанюк Денис Сергеевич / Denis S. Godanyuk; e-mail: godanyuk@gmail.com

Рукопись поступила/Received: 18.05.2018. Принята в печать/Accepted for publication: 15.08.2018. 


\begin{abstract}
Fracture and migration of metal implants is a well-known issue which is especially relevant for actively loaded zones with a high amplitude of physiological movements. The authors analyzed 17 publications dedicated to Kirschner wire migration into the spinal canal after fixation of acromioclavicular joint (ACJ) injury. The present paper contains literature review and own clinical case of the authors. The authors generalize the conceptions of migration causes, surgical tactics and prevention recommendations. The key reason of fracture and migration of Kirschner wires during fixation of ACJ injury is the instability of implants, trans-articular wire insertion during fixation of reduced dislocation of acromial end of the clavicle, insufficient immobilization and untimely implants removal after removal of immobilization. Implants migration into the spinal canal is the indication for their surgical removal irrespective of clinical signs. In the majority of studied publications authors described posterior approach or lateral approach aligned with the migration direction. No grafting techniques for dura mater defects were present in the studied literature. The authors of the current paper justify a surgical procedure for removal of migrated implant using a combined posterior and lateral approach on the own clinical case. The choice of procedure algorithm results from the need for prophylaxis of secondary spinal cord lesion and liquorrhea during removal of migrated implants from spinal canal.
\end{abstract}

Keywords: Kirschner wire migration, spinal cord lesion, liquorrhea, acromioclavicular joint, clavicle fracture.

Competing interests: the authors declare that they have no competing interests.

Funding: the authors have no support or funding to report.

Consent for publication: the patient provided voluntary consent for publication of case data.

\section{Введение}

Перелом и миграция элементов металлоконструкций является грозным осложнением остеосинтеза, приобретающим особую опасность при их смещении в смежные анатомические зоны. Переломы спиц Киршнера и других фиксирующих имплантатов с их локальным смещением нередко регистрируются даже при их первичной корректной установке [1].

Механизм миграции элементов конструкций, используемых для фиксации повреждений акромиально-ключичного сочленения, до конца не ясен. В качестве наиболее вероятных причин называют действие разнонаправленных кинетических сил, воздействующих на акромиальный конец ключицы, сокращения мышц, дыхательные движения, отрицательное внутригрудное давление на вдохе, гравитационные силы и локальную резорбцию кости вокруг имплантата [2-7].

Описаны случаи повреждения имплантатами пищевода, трахеи, магистральных сосудов шеи, легких, аорты и сердца у пациентов, ранее оперированных по поводу повреждений ключицы и акромиально-ключичного сустава, в том числе с летальными исходами [3, 8-12].

Миграция фиксирующих элементов в позвоночный канал сопряжена с риском возникновения грубого неврологического дефицита [5, 7, 13, 14], а также повреждения спинного мозга, крупных сосудов шеи и плевры в ходе их хирургического удаления $[14,15]$.

Цель сообщения - обратить внимание коллег на возможность развития редкого осложнения фиксации акромиально-ключичного сочленения миграцию фрагмента имплантата в позвоночный канал с повреждением твердой мозговой оболочки и корешков спинного мозга.

\section{Обзор литературы}

В доступной литературе мы нашли 17 сообщений, посвященных миграции имплантатов в позвоночный канал у 18 пациентов, ранее перенесших фиксацию повреждений акромиально-ключичного сочленения и ключицы спицами Киршнера. Поиск выполняли в базах данных PubMed, eLIBRARY. Изучены сообщения за период с 2006 по 2018 г., более ранние сообщения цитированы. Критерием включения послужило описание миграции фрагмента спицы в позвоночный канал после фиксации повреждений акромиально-ключичного сочленения и ключицы. Сообщения, посвященные миграции имплантатов после других видов спицевых фиксирующих операций и в другие анатомические зоны, в обзор на включались.

В изученных нами сообщениях возраст пациентов варьирует от 22 до 72 лет, преобладают пациенты среднего возраста. В 11 сообщениях пациентами были мужчины, в 2 - женщины, в 4 сообщениях пол пациента не указан. Длительность периода между стабилизирующей операцией и миграцией конструкции составила от 11 суток до 12 лет. В 11 случаях миграция произошла в течение первого года после операции, из них в 7 случаях - в сроки от 2 до 6 мес. Во всех случаях спица входила в позвоночный канал через корешковые отверстия на уровнях от C5-C6 до Th2-Th3, причем в 9 наблюдениях на уровне C7-Th1. В 7 сообщениях из 17 пациенты были оперированы справа (в 10 не установлено).

Указание на причины миграции элементов конструкции приводится в 6 сообщениях. Во всех случаях миграция сопровождалась нестабильностью фиксации в виде резорбции кости или перелома спицы. С.В. Сиваконь с соавторами [16] считают возможными причинами нестабильности трансартикулярное проведение спиц, недостаточность 
иммобилизации, а также несвоевременное удаление конструкций после снятия иммобилизации. В качестве других вероятных причин миграции спиц указываются особенности анатомии и биомеханики верхнего плечевого пояса, недостаточная иммобилизация руки, отрицательное давление в грудной полости на вдохе, падения, особенности конституции больных (ожирение в одном наблюдении), повторные травмы (падения) и нагрузка на оперированную руку.

Клиническая картина в 4 сообщениях была представлена признаками поражения спинного мозга в виде нижнего парапареза, тетрапареза или синдрома Броун-Секара [5, 7, 15, 17]. Корешковая боль, соответствующая уровню вхождения спицы в позвоночный канал, описана в 3 наблюдениях [4, 13, 18]. В двух случаях клиника была представлена признаками повреждения легких и плевры (эмфизема и пневмоторакс) без неврологического дефицита $[19,20]$. В наблюдении L. Minić с соавторами в качестве ведущего клинического проявления описан синдром ликворной гипотензии [13]. Бессимптомная миграция фрагмента спицы Киршнера в позвоночный канал описана S. Bennis с соавторами [21].

В большинстве наблюдений (10 сообщений) для удаления инородного тела из позвоночного канала использованы латеральные подходы (надключичный, торакотомия) к позвоночнику, обеспечивающие возможность удаления спицы в направлении её оси. Подобная тактика использовалась как у пациентов без признаков поражения спинного мозга и его корешков, так и у пациентов с грубой неврологической симптоматикой [7, 17]. M.А. Никольский с соавторами и P. Fransen с соавторами использовали комбинацию надключичного и заднего доступов в позвоночный канал для мобилизации спицы в латеральном направлении и ее контроля при удалении [7, 14]. В сообщении P. Fransen с соавторами подробно обсуждаются преимущества комбинации доступов. В частности, указывается на возможность контроля интрадурального гемостаза, герметизации твердой мозговой оболочки и предупреждения смещений дистального конца спицы при ее удалении [14]. Аналогичные преимущества контроля со стороны позвоночного канала приводит W. Mamane с соавторами [19].

В двух наблюдениях описано истечение ликвора из дефекта TMO, наблюдавшееся во время операции [20, 21]. Послеоперационная ликворея не упоминается ни в одном наблюдении. В то же время, использованный способ герметизации твердой мозговой оболочки в указанных сообщениях не описывается. Ряд авторов, обсуждая тактику операций, указывают на возможность контроля ликворостаза из доступов через позвоночный канал $[14,19]$. Симптомокомплекс, напоминающй ликворную гипотензию, описан в качестве до- минирующего клинического синдрома в статье L. Minić с соавторами. Примечательно, что интраоперационно при удалении спицы через трансторакальный доступ, признаков ликвореи не выявлено [13]. L. Yawei с соавторами отметили истечение ликвора по раневому каналу при удалении спицы из торакоскопического доступа. Признаков ликворной гипотензии и ликвореи ни до, ни после операции не описано, пластика ликворной фистулы не выполнялась [20].

В цитируемых работах авторами указаны различные способы предупреждения миграции элементов конструкций. Среди них есть рекомендации технического и организационного характера.

Большинство авторов указывает на необходимость информирования травматологов и хирургов о возможности подобных осложнений. В некоторых сообщениях особо подчеркивается важность информирования пациентов о возможности перелома и миграции конструкции $[6,14,18]$. Регулярный рентгеновский контроль с интервалами в 2-4 нед. и удаление конструкции после консолидации зоны повреждения или при появлении признаков нестабильности считают обязательным большинство авторов. Многие рекомендуют удалять спицы через 6-8 нед. после операции $[5,6,14]$. На время фиксации обязательны строгая иммобилизация зоны повреждения [19] и исключение отведений оперированной руки выше $90^{\circ}[5,6]$. С.В. Сиваконь с соавторами считают необходимым удаление конструкции до начала реабилитационых процедур и восстановления активных движений [16].

J. Liberski акцентирует внимание на необходимости оценки степени физической активности, способности пациента к самообслуживанию и выполнению рекомендаций. Автор рекомендует делать выбор в пользу других способов фиксации у спортсменов и людей, ведущих активный образ жизни, у пожилых и нуждающихся в посторонней помощи людей, а также у непунктуальных пациентов (злоупотребление алкоголем, прием наркотиков, интеллектуальные нарушения) [22]. Также ограничить показания к остеосинтезу спицами при повреждениях ключицы и акромиально-ключичного сочленения рекомендует М.А. Никольский с соавторами [7].

Некоторые авторы, в частности P. Fransen указывают на повышение надежности фиксации при сгибании дистального конца спицы под углом 90 [14]. L. Yawei считает, что остеосинтез спицами повреждений ключицы и акромиально-ключичного сочленения следует признать очень опасной манипуляцией. В сообщении подчеркивается возможность миграции всех типов спиц - гладких, с резьбой и изогнутых на конце [20]. S. Bennis также указывает на недостаточную надежность фиксации спицами и считает остеосинтез пластинами более безопасной 
операцией с меньшей частотой несращений и риском миграции [21].

\section{Клиническое наблюдение}

Пациенту 64 лет в 1997 г. по поводу повреждения правого акромиально-ключичного сочленения была выполнена фиксация спицами Киршнера. Спустя два года, после работы в гараже (ремонт автомобиля в положении лежа на спине) пациент заметил появление боли и кровоподтека в области правого плечевого сустава. Спустя еще год появились боли в области шеи с иррадиацией в правую руку. За медицинской помощью он не обращался. При плановом медосмотре в 2016 г. на флюорограмме было выявлено инородное тело металлической плотности в проекции позвоночника.

По данным КТ, в позвоночном канале на уровне C6-C7 определялось инородное тело (спица) длиной 4,5 cм, располагающееся косо в передней трети позвоночного канала, проксимальный конец на уровне корня дужки С7 слева, дистальный конец в правом корешковом отверстии С6-С7 на уровне наружного края суставных фасеток (рис. 1).

При поступлении в клинику пациент предъявлял жалобы на локальную боль в области шеи с иррадиацией по медиальной поверхности правого предплечья и кисти, усиливающуюся при натуживании и кашле. Неврологический статус представлен корешковым болевым синдромом С8 справа. Общемозговой, проводниковой и менингеальной симптоматики не было.
3.05.2017 выполнена операция: удаление интрадурального инородного тела (фрагмента спицы Киршнера) на уровне C7-Th1 из комбинированного доступа, пластика дефекта твердой мозговой оболочки.

Под общим обезболиванием, в положении пациента на левом боку, выполнен боковой надключичный подход к 1-му шейному ребру справа между трапециевидной и задней лестничной мышцами (рис. 2 a). Над поперечным отростком Th1 обнаружен нерв C8, выходящий из корешкового отверстия C7-Th1, уровень манипуляций верифицирован при помощи ЭОП. Визуально и пальпаторно инородных тел в зоне манипуляций не выявлено (рис. 2 b).

Выполнен разрез кожи и подкожной клетчатки в проекции остистых отростков C6-Th1, скелетированы остистый отросток и пластинчатая часть дуги С7 справа, медиальная часть сустава C7-Th1. Выполнена флавэктомия, медиальная фасетэктомия C7-Th1 справа. В позвоночном канале визуализирован фрагмент спицы Киршнера, перфорирующий ТМО выше корешка С8 (рис. 2 c). После мобилизации эпидуральных вен и корешка C8, спица смещена в правое корешковое отверстие и удалена из надключичного доступа (рис. 2 d). Из дефекта твердой мозговой оболочки в рану поступал прозрачный бесцветный ликвор. Выполнено замещение дефекта ТМО пластинами Тахокомб. Гемостаз состоятелен при АД 130/90 мм рт. ст. Проба Вальсальвы - ликвореи нет. Раны ушиты послойно без дренажей.
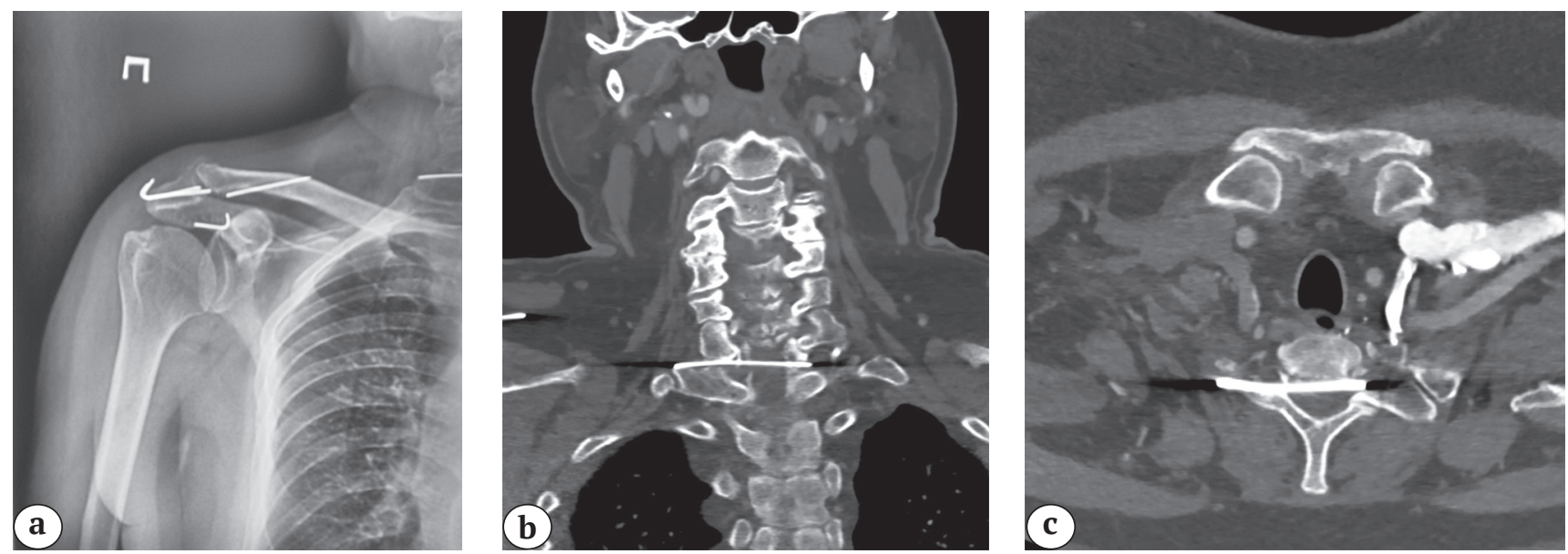

Рис. 1. Предоперационная рентгенограмма и КТ шейного отдела позвоночника пациента:

a - рентгенограмма правого плечевого сустава - состояние после фиксации акромиально-ключичного сочленения спицами, определяется перелом фиксирующих имплантов, дистальный фрагмент одной из спиц визуализируется в проекции тела С7 позвонка;

б - КТ шейного отдела позвоночника, коронарная проекция: в позвоночном канале на уровне тела С7 позвонка определяется инородное тело металлической плотности, проникающее через правое корешковое отверстие С6-С7; с - аксиальная проекция - определяется металлическая спица в позвоночном канале

Fig. 1. Preoperative cervical spine X-ray images:

a - X-ray images of the right shoulder after acromioclavicular fixation with K-wire, the disruption

of one of the spokes is seen, distal part of other spoke located at the level of C7;

$\mathrm{b}-\mathrm{CT}$-scan of the cervical spine, there is foreign body of metal density, penetrating right radicular foramen C6-C7;

$\mathrm{c}$ - axial plane - K-wire in the spine canal 

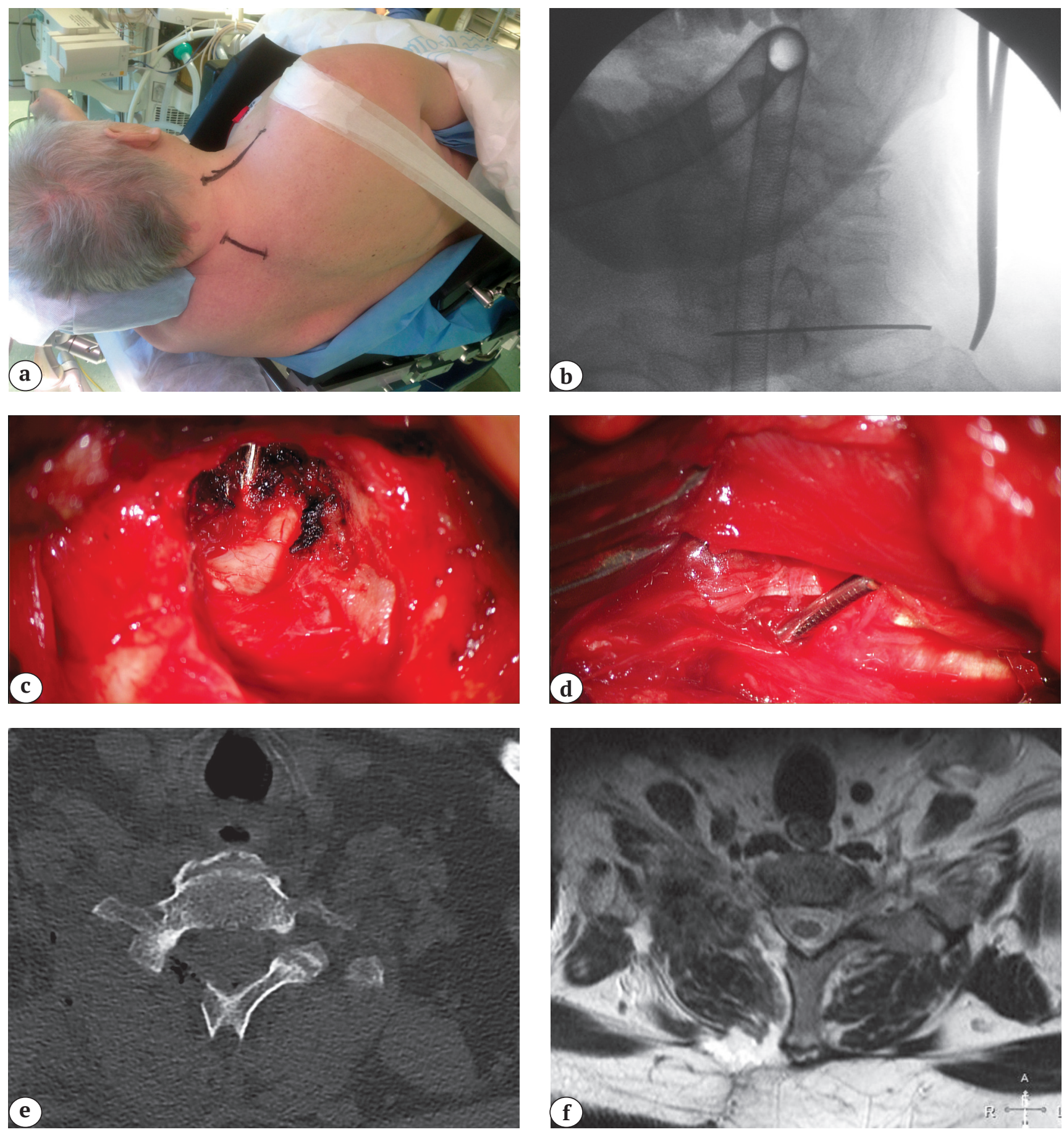

Рис. 2. Этапы операции и результаты лучевого исследования:

a - положение пациента на операционном столе - на левом боку, намечены правосторонний

надключичный и задний срединный доступы; b - интраоперационный рентгеновский контроль в ходе выполнения надключичного доступа - зона манипуляций соответствует уровню C7-Th1;

c - выполнены интерламинэктомия, медиальная фасетотомия справа на уровне C7-Th1, визуализирована спица, перфорирующая ТМО на уровне корешка C8; d - смещенная в латеральном направлении спица видна на уровне выхода из корешкового отверстия C7-Th1; е - КТ после операции; f - MPT после операции

Fig. 2. Procedure stages and postoperative X-ray control:

a - patient positioning on surgical table - lateral decubitus, marked right supraclavicular and posterior median approaches; $\mathrm{b}$ - intraoperative X-ray control during supraclavicular approach - manipulation area corresponds to level C7-Th1; c - interlaminectomy, medial facetectomy on the right at level C7-Th1, wire is visualized, dura mater perforated at the level $\mathrm{C} 8$; $\mathrm{d}$ - wire displaced in lateral director is seen at the level of exit from radicular foramen of C7-Th1; e - CT after the surgery; $\mathrm{f}$ - MRI after the surgery 
Послеоперационный период протекал без осложнений, раны зажили первично, пациент выписан из стационара на 6-е сутки после операции. На контрольном осмотре через 1,5 мес. после операции рубцы без признаков воспаления, в неврологическом статусе регресс корешкового болевого синдрома. Пациент направлен к травматологу для удаления оставшихся элементов конструкции в области правого акромиально-ключичного сочленения.

\section{Обоснование хирургической тактики}

Возможность сокращения рисков ятрогенного повреждения спинного мозга и послеоперационных осложнений при сочетании латерального и заднего подходов обусловило выбор принятой нами хирургической тактики. Основой принятого плана явилась оценка рисков ключевых этапов операции - визуализации, мобилизации и удаления инородного тела в позвоночном канале. Незначительное выступание проксимального конца спицы из корешкового канала и его изгиб создавали опасность отклонения дистального конца имплантата с повреждением нервной ткани при его мобилизации, фиксации и тракции. Таким образом, для предупреждения вторичного травмирования спинного мозга во время основных этапов операции необходим контроль положения дистального конца спицы в позвоночном канале. Возможность ранения крупных сосудов шеи при мобилизации спицы латерально от позвоночника требует предварительной визуализации зоны выхода из корешкового канала C7-Th1.

В нашем наблюдении имел место точечный дефект твердой оболочки, расположенный на боковой ее поверхности выше отхождения корешка, что обусловило выбор способа пластики.

\section{Обсуждение}

Миграция фрагментов спиц Киршнера в позвоночный канал является одним из возможных осложнений при фиксации повреждений акромиально-ключичного сочленения с высокой частотой развития неврологического дефицита $[4,5,7,13,15$, $17,18]$. Перелом спицы как следствие нестабильности фиксации является основной причиной данного осложнения и требует пристального внимания со стороны хирурга и пациента в течение всего времени стояния конструкции [6, 7, 15, 18, 20, 21].

Наличие данного осложнения является показанием к хирургическому лечению с целью предупреждения прогрессирования неврологического дефицита и дальнейшей миграции [7, 15, 19]. Нам представляется обоснованным использование комбинации латеральных и задних доступов во всех случаях интраканального расположения мигрировавшего имплантата, вне зависимости от сте- пени выраженности неврологического дефицита. Задачами этапа, выполняемого через позвоночный канал, в таких случаях являются профилактика повреждений спинного мозга при удалении спицы, мобилизация ее в латеральном направлении, контроль интрадурального гемостаза и профилактика ликвореи. Возможно, описанное в наблюдениях L. Yawei с соавторами и M. Ljubodrag с соавторами $[13,20]$ купирование ликвореи без дополнительных ликворостатичеких манипуляций при удалении спицы через боковой торакотомный доступ объясняется точечным дефектом TMO и узким раневым каналом в паравертебральных мягких тканях. В то же время, по нашему мнению, сообщение ликворопроводящих путей с естественными полостями организма или хирургической раной всегда потенциально опасно развитием ликвореи и требует тщательной герметизации дефекта, а также изоляции зоны пластики TMO от естественных и ятрогенных полостей.

Фиксация акромиально-ключичного сочленения спицами Киршнера сопряжена с риском инвалидизирующих и летальных осложнений $[5,7$, $12-15]$. Вероятность развития жизнеугрожающих состояний, обусловленных миграцией фиксирующих элементов, заставляет считать метод недостаточно надежным и безопасным.

Этика публикации: пациент дал добровольное информированное согласие на публикацию клинического наблюдения.

\section{Конфликт интересов: не заявлен.}

Источник финансирования: исследование проведено без спонсорской поддержки.

\section{Литература [References]}

1. Медведчиков А.Е., Жиленко В.Ю., Свешников П.Г., Буров Е.В. Анализ результатов лечения больных с повреждением акромиально-ключичного сустава. Модифицированный метод ревизионного лечения пациентов с рецидивировавшими вывихами акромиального конца ключицы. Современные проблемы науки и образования. 2015;6. Режим доступа: https:// www.science-education.ru/ru/article/view?id=23260.

Medvedchikov A.E., Ghilenko V.Yu., Sveshnikov P.G., Burov E.V. [Analysis of results of treatment of patients with clavicle-acromialis joint trauma. The modified method of treatment patients with recurrent luxation of clavicle`s acromial end]. Sovremennye problemy nauki i obrazovaniya [Modern Problems of Science and Education]. 2015;6. (In Russ.). Available from: https:// www.science-education.ru/ru/article/view?id=23260.

2. Булычев Г.И., Г.А. Блувштейн. Выбор способа хирургического лечения больных с вывихом акромиального конца ключицы. Гений ортопедии. 2002;(3):46-48. Bulychev G.I., G.A. Bluvshtejn [The choice of surgical treatment of patients with luxation of clavicle`s acromial end]. Genij Ortopedii [Orthopaedic Genius]. 2002;(3):4648. (In Russ.).

3. Сиротко В.В., Никольский М.А., Железняк А.В., Подолинский С.Г., Бейнер Ю.Ф. Миграция спицы 
в заднее средостение после остеосинтеза акромиально-ключичного сочленения. Новости хирургии. 2010;18(2):133-136.

Sirotko V.V., Nikolsky M.A., Jeleznyak A.V., Podolinsky S.G., Beiner Yu.F. [Migration of the knitting needle to the posterior mediastinum after osteosynthesis of the acromioclavicular articulation]. Novosti hirurgii 2010;18(2):133-136. (In Russ.).

4. Lee J.H., Chung J.Y., Kim M.S. Unusual migration of kirschner's wire into intervertebral foramen after lateral clavicle fracture fixation - a case report. Clin Shoulder Elbow. 2014;17(2):77-79.DOI : 10.5397/cise.2014.17.2.77.

5. Regel J.P., Pospiech J., Aalders T.A., Ruchholtz S. Intraspinal migration of a Kirschner wire 3 months after clavicular fracture fixation. Neurosurg Rev. 2002;25(1-2):110-112.

6. Tsai C.H., Hsu1 H.C., Huan C.Y., Chen H.T., Fong Y.C. Late Migration of Threaded Wire (Schanz Screw) from Right Distal Clavicle to the Cervical Spine. I Chin Med Assoc. 2009;72(1):48-51. DOI: 10.1016/S1726-4901(09)70021-8.

7. Никольский М.А., Протас Р.Н., Кубраков К.М. О возможных осложнениях металлоостеосинтеза при переломах проксимального конца плечевой кости. Вестник Витебского государственного медицинского университета. 2006;5(1):110-113.

Nikolsky M.A., Protas R.N., Kubrakov K.M. [About possible complications of metalloesteosynthesis with fractures of the proximal end of the humerus]. Vestnik Vitebskogo gosudarstvennogo meditsinskogo universiteta [Vestnik of Vitebsk State Medical University]. 2006;5(1):110-113. (In Russ.).

8. Sharma H., Taylor G., Clarke N. A review of k-wire related complications in the emergency management of paediatric upper extremity trauma. Ann R Coll Surg Engl. 2007;89(3):252-258. DOI: 10.1308/003588407X155482.

9. Foster G.T., Chetty K.G., Mahutte K., Kim J.D., Sasse S.A. Hemoptysis due to migration of a fractured kirschner wire. Chest. 2001;119(4):1285-1286.

10. Jung G.H., Kim T.H., Cho H.I. Granulation tissue formed by stimulating k-wire mimicking tuberculous cervical lymphadenopathy: a case report. J Korean Fract Soc. 2014;27(3):227-231.

11. Mankowski B., Polchlopek T., Strojny M., Grala P., Slowinski K. Intraspinal migration of a Kirschner wire as a late complication of acromioclavicular joint repair: a case report. I Med Case Rep. 2016;10:66. DOI: $10.1186 / \mathrm{s} 13256-016-0844-4$.

12. Ram G.G., Vijayaraghavan P.V. K wire: a lethal implant. Int J Sci Rep. 2015;1(1):83-85.

\section{СВЕДЕНИЯ ОБ АВТОРАХ}

Гуляев Дмитрий Александрович - д-р мед. наук, профессор, научный руководитель отделения нейрохирургии № 5, ФГБУ «Национальный медицинский исследовательский центр им. В.А. Алмазова» Минздрава России, Санкт-Петербург

Годанюк Денис Сергеевич - врач-нейрохирург отделения нейрохирургии № 5, ФГБУ «Национальный медицинский исследовательский центр им. В.А. Алмазова» Минздрава России, Санкт-Петербург

Каурова Татьяна Анатольевна - канд. мед. наук, врач-нейрохирург отделения нейрохирургии № 5 , ФГБУ «Национальный медицинский исследовательский центр им. В.А. Алмазова» Минздрава России, Санкт-Петербург
13. Minić L., Lepić M., Novaković N., Mandić-Rajčević S. Symptomatic migration of a Kirschner wire into the spinal canal without spinal cord injury: case report. I Neurosurg Spine. 2016;24:291-294. DOI: $10.3171 / 2015.5$.SPINE1596.

14. Fransen P., Bourgeois S., Rommens J. Kirshner wire migration causing spinal cord injury one year after internal fixation of a clavicle fracture. Acta Orthop Belg. 2007;73(3):390-392.

15. Wang Z., Liu Y., Ou Z., Leng J., Fu C., Liu G. Penetrating injury of the spinal cord treated surgically. Orthopedics. 2012;35:1136-1140. DOI: 10.3928/01477447-20120621-41.

16. Сиваконь С.В., Девин И. В., Кибиткин А. С., Абдуллаев А.К., Моисеенко В.А. Казуистический случай миграции ятрогенного инородного тела. Известия высших учебных заведений. Поволжский регион. Медицинские науки. 2012;1(21):85-89.

Sivakon S.V., Devin I.V., Kibitkin A.S., Abdullaev A.K., Mouseenko V.A. [Casuistic case of migration of an iatrogenous foreign body]. Izvestiya vysshikh uchebnykh zavedenii. Povolzhskii region. Meditsinskie nauki. [News of Higher Educational Institutions. The Volga Region. Medical Sciences]. 2012;1(21):85-89. (In Russ.).

17. Loncán L.I., Sempere D.F., Ajuria J.E. BrownSequard syndrome caused by a Kirschner wire as a complication of clavicular osteosynthesis. Spinal Cord. 1998;36(11):797-799.

18. Gonsales D., Aguilar-Salinas P., Cavicchioli A., Felicio A., Bastos L.F., Nicandro F. The migration of kirschner wire from left distal clavicle to the intradural anterior thoracic spine. OAJNN. 2017;2(4):14 DOI: 10.19080/OAJNN.2017.02.555595.

19. Mamane W., Breitel D., Lenoir T., Guigui P. [Spinal migration of a Kirschner wire after surgery for clavicular nonunion. A case report and review of the literature]. Chir Main. 2009;28(6):367-369. (in French). DOI: 10.1016/j.main.2009.08.007.

20. Yawei L., Wang B., Guohua L., Guangzhong X., Weidong L. Video-assisted thoracoscopic surgery for migration of a kirschner wire in the spinal canal: a case report and literature review. Turk Neurosurg. 2013;23(6): 803-806. DOI: 10.5137/1019-5149.JTN.5300-11.1.

21. Bennis S., Scarone P., Lepeintre J.F., Puyo P. Aldea S., Gaillard S. Asymptomatic spinal canal migration of clavicular K-wire at the cervicothoracic junction. Orthopedics. 2008;31(12). pii: orthosupersite. com/view.asp?rID=32939.

22. Liberski J., Ficek K. Kirschner wire migration from the clavicle to the cervical spine. Int J Case Reports Images. 2013;4(6):308-311. DOI: 10.5348/ijcri-2013-06-319-CR-4.

\section{INFORMATION ABOUT AUTHORS}

Dmitry A. Gulyaev - Dr. Sci. (Med.), professor, chief of the Department of Neurosurgery N 5, Almazov National Medical Research Centre, St. Petersburg, Russian Federation

Denis S. Godanyuk - neurosurgeon, Department of Neurosurgery N 5, Almazov National Medical Research Centre, St. Petersburg, Russian Federation

Tatiana A. Kaurova - Cand. Sci. (Med.), neurosurgeon, Department of Neurosurgery N 5, Almazov National Medical Research Centre, St. Petersburg, Russian Federation 
Красношлык Павел Владимирович - канд. мед. наук, заведующий отделения нейрохирургии № 5, ФГБУ «Национальный медицинский исследовательский центр им. В.А. Алмазова» Минздрава России, Санкт-Петербург

Майков Сергей Валерьевич - канд. мед. наук, младший научный сотрудник отделения спортивной травматологии и реабилитации, ФГБУ «Российский научноисследовательский институт травматологии и ортопедии им. Р.Р. Вредена» Минздрава России, Санкт-Петербург
Pavel V. Krasnoshlyk - Cand. Sci. (Med.), head of the Department of Neurosurgery N 5, Almazov National Medical Research Centre, St. Petersburg, Russian Federation

Sergey V. Maikov - Cand. Sci. (Med.), researcher, Department of Sports Traumatology and Rehabilitation, Vreden Russian Research Institute of Traumatology and Orthopedics, St. Petersburg, Russian Federation 